\title{
Introduction to the symposium on discontinuous games
}

\author{
Philip J. Reny ${ }^{1}$
}

Received: 18 February 2016 / Accepted: 20 February 2016 / Published online: 14 March 2016 (C) Springer-Verlag Berlin Heidelberg 2016

The literature on the existence of Nash equilibrium in discontinuous games has blossomed since the seminal contribution of Dasgupta and Maskin (1986). The present symposium brings together a number of recent developments. Throughout this introduction, it is assumed that any game $G=\left(X_{i}, u_{i}\right)_{i \in N}$ under consideration has a finite player set, $N$, that each player $i \in N$ has a nonempty, compact, and convex set of pure strategies $X_{i}$ that is a subset of a linear topological space, and has bounded payoff function $u_{i}: X \rightarrow \mathbb{R}$, where $X=\times_{i \in N} X_{i}$. Not all papers in the symposium are always so restrictive as this. Indeed, some authors occasionally do not require strategy sets to be convex nor do they always require the existence of utility representations of the players' preferences over strategy profiles. Such exceptions will be noted in this introduction only when absolutely necessary. Also, "Nash equilibrium" will always mean pure strategy Nash equilibrium. Each paper included in this symposium issue is briefly discussed below, and the papers have been arranged in alphabetical order.

The paper by Guilherme Carmona (2014) entitled "Reducible equilibrium properties: comments on recent existence results," is focused on connecting a rather wide variety of existence results in the literature by way of a common proof technique. Carmona's paper shows that the various sufficient conditions for existence can all be understood as allowing the existence problem to be reduced to a "simpler" existence problem in which the relevant best-reply correspondences are well behaved, i.e., upper hemicontinuous, nonempty valued, and convex valued, on a domain that is amenable to fixed point analysis, i.e., nonempty, compact and convex. While some early results in the literature take a related route (e.g., Dasgupta and Maskin 1986; Reny 1999, both first reduce the problem to a game setting in which standard existence results

Philip J. Reny

preny@uchicago.edu

1 Department of Economics, University of Chicago, Chicago, IL, USA 
can be applied), the recent literature's more sophisticated techniques are rather further removed from such a reduction principle, although, in the end, one cannot get away from an eventual application of Kakutani-Fan-Glicksberg. In any case, the unifying perspective offered here is both welcome and illuminating.

The paper by Guilherme Carmona and Konrad Podczeck (2015) entitled, "Existence of Nash equilibrium in ordinal games with discontinuous preferences," provides several new Nash equilibrium existence results for discontinuous games that generalize many in the literature, including the main result in the paper by Reny included in this symposium issue. The authors introduce the notions of target point security and target correspondence security which generalize Reny's point security and correspondence security conditions, respectively. The authors allow the players' preferences to be given by binary relations that need not be complete or transitive. This can be especially fruitful when preferences are those of a group of individuals. Another difference between point security and target security is, very roughly, that when a strategy profile, $x$, is not a Nash equilibrium, point security requires the existence of a deviation strategy profile $\hat{x}$ and a neighborhood of $x$ such that, for every strategy $y$ in the neighborhood, some player $i$ has a strictly profitable deviation to $\hat{x}_{i}$, even when, in reaction, the others deviate to their parts of any $x^{\prime}$ in the neighborhood. Target security, very roughly, replaces the strategy profile $y$ that is "targeted" for improvement, with some other strategy profile, the choice of which is flexible. The target security conditions are very permissive and subtle. Taking full advantage of their flexibility may sometimes require one to patch together a number of local conditions so that they "fit together" across the entire strategy space. While non-local conditions of this sort are not as easy to verify as purely local conditions, one should view the non-local nature of the conditions here as a useful option, since there are free parameters (the players' "target functions") that can be set so that the conditions become local. Carmona and Podczeck give several applications of their results, including a very general result on the existence of equilibria in abstract games. This appears to be the best result of its kind to date.

The paper by János Flesch and Arkadi Predtetchinski (2015) entitled, "Subgameperfect epsilon-equilibria in perfect information games with sigma-discrete discontinuities," is the only paper in this symposium to consider dynamic games with discontinuous payoffs. Here, the objective is to begin to understand, in the context of perfect information games with infinitely many actions, the structure of the sets of plays, i.e., infinite sequences of actions, on which discontinuities may be permitted without precluding the existence of a subgame perfect epsilon equilibrium. The setup is wonderfully crisp. Action spaces at each date are given the discrete metric, and the set of infinite sequences of plays is given the product topology. In particular, no compactness is assumed whatsoever. The key concept is that of a "discrete" set. A subset $D$ of a topological space is discrete if every point in $D$ is contained in an open set whose intersection with $D$ contains only the point itself. In many spaces of interest, e.g., Polish spaces, a set is discrete only if it is countable. But that is not so here. Indeed, there are discrete sets of plays that are uncountable. For example, consider the perfect information game in which two players alternately choose any real number forever. The set of plays is therefore the set of all infinite sequences of real numbers. The subset of plays consisting of all sequences that are eventually constant is a discrete 
set with a continuum of elements in the product topology that we have defined. Flesch and Predtetchinski prove the following intriguing theorem. Suppose that all of the players' payoff functions are bounded. If, in addition, the players' payoff functions are all continuous on a set of plays outside a discrete set, then, for any $\varepsilon>0$, the game of perfect information has at least one subgame perfect $\varepsilon$-equilibrium. Furthermore, no player need mix over more than a countable number of actions whenever it is his turn to play. Interestingly, mixing is necessary for the result and its role is not entirely unfamiliar in the context of discontinuous games. Players mix, not to make opponents indifferent (such indifference should not be necessary given perfect information and no moves of nature), but, rather, in order to make opponents' payoff functions continuous. A related approach can be traced back to at least the seminal paper by Dasgupta and Maskin (1986), whose ideas can also be found in Reny (2010). In each of these instances, the idea is that if discontinuities require certain coincidences of actions to occur, then adding a small amount of noise (here via mixed strategies) can render those coincidences and hence also the discontinuities they generate, impossibly unlikely or nearly so.

The paper by Wei He and Nicholas Yannelis (2015) entitled, "Existence of Walrasian equilibria with discontinuous, non-ordered, interdependent and price-dependent preferences," provides an impressive generalization of Shafer and Sonnenschein's (1975) equilibrium existence result in abstract economies. The objective is to permit discontinuities in the players' preference relations, as might occur, for example, in an auction setting, Bertrand price competition, or in a competitive economy with fixed costs of production. The concept of majorization of a correspondence via the patching together of locally defined correspondences plays a key role here, as in the Prokopovych paper that is discussed below. He and Yannelis say that a correspondence, $F$, has the continuous inclusion property at a point in its domain if, on a neighborhood of the point, one can define a correspondence whose values are contained in the values of $F$ and whose convexified values yield a correspondence with a closed graph. The key assumption in He and Yannelis' generalization of Shafer and Sonnenschein (1975) is that, for each player, the correspondence that is defined by the intersection of his feasibility correspondence and his strict preference correspondence has the continuous inclusion property. A related, but slightly different condition can be found in Reny (2015b). The existence theorem for abstract economies obtained here by He and Yannelis is among the strongest in the literature.

The paper by Rabia Nessah and Guoqiang Tian (2015) entitled, "On the existence of Nash equilibrium in discontinuous games," introduces several new definitions and conditions that permit extensions of many of the Nash equilibrium existence results currently in the literature, including several in this symposium issue. A first new condition that is introduced here is that of a quasi-weakly transfer continuous game. To understand this new condition, let us first define for any strategy profile, $x$, player $i$ 's lower-envelope payoff, $\underline{u}_{i}(x)$, to be the smallest limiting payoff that player $i$ can receive along any sequence of strategies converging to $x$ in which player $i$ does not deviate from his strategy in $x$, that is, $\underline{u}_{i}(x)=\liminf _{x^{\prime} \rightarrow x} u_{i}\left(x_{i}, x_{-i}^{\prime}\right)$. A game is said to be quasi-weakly transfer continuous if whenever a strategy profile, $x$, is not a Nash equilibrium some player $i$ has a deviation strategy, $y_{i}$, such that $\underline{u}_{i}\left(y_{i}, x_{-i}^{\prime}\right)>\underline{u}_{i}\left(x^{\prime}\right)$ 
holds for every $x^{\prime}$ in some neighborhood of $x .^{1}$ The lower-envelope payoff function was first introduced in Simon (1987) and its use there inspired its heavy use in Reny (1999) and in many other studies of discontinuous games since. Nessah and Tian's Theorem 1 states that under standard conditions, if a game is quasi-weakly transfer continuous, then it possesses a Nash equilibrium. There are several reasons that the lower-envelope payoff function is especially useful. First, it is lower semicontinuous in the other players' strategies for any given strategy of the owner. Second, it inherits quasi-concavity in the owner's strategy from the original payoff function. Third, if the game is quasi-weakly transfer continuous, then any Nash equilibrium of the game in which all the players' payoff functions are replaced with their lower-envelope payoff functions is a Nash equilibrium of the original game. Thus, the problem of establishing the existence of a Nash equilibrium in a game with discontinuous payoffs can be reduced to a problem in which each players' payoff function is lower semicontinuous in the others' strategies. There is more work to do to prove the existence of a Nash equilibrium, but this additional structure is quite helpful. Nessah and Tian provide numerous other interesting extensions and generalizations of results in the literature, including results that relax the own-strategy quasi-concavity assumption. There, the techniques are closely related to those first developed in the beautiful and important paper of Baye et al. (1993).

The paper by Pavlo Prokopovych (2015) entitled, "Majorized correspondences and equilibrium existence in discontinuous games," provides conditions that allow one to patch together locally defined correspondences with possibly empty values on a domain that is nonempty, compact, and convex (as in, for example, the work of McLennan et al. 2011; Barelli and Meneghel 2013) to obtain a globally defined correspondence, $F$ say, that maps into convex subsets of the domain, is upper hemicontinuous, yet fails to have a fixed point. By the Kakutani-Fan-Glicksberg fixed point theorem, any such correspondence $F$ must be somewhere empty valued. When the domain, $X$ say, is the set of strategy profiles in a game, and, for any $x \in X$, the value $F(x)$ is the set of strategy profiles whose $i$-th coordinate is a strictly profitable unilateral deviation for player $i$ against $x$, then any $x$ for which $F(x)$ is empty is an equilibrium of the game (see, e.g., Debreu 1952; Shafer and Sonnenschein 1975). Prokopovych observes that it suffices to construct via this patching technique, a correspondence $G$ that majorizes $F$, i.e., $G(x)$ contains $F(x)$ for every $x$, and he provides conditions under which such a majorization $G$ must be somewhere empty valued. Propkopvych gives several applications of his results that either supplement our understanding of known existence results or further extend some those results.

The paper by Philip Reny (2015a) entitled, "Nash equilibrium in discontinuous games," provides a new result on the existence of Nash equilibrium that is entirely ordinal, a property that should be expected of any result on the existence of pure strategy Nash equilibrium. Even so, the hypotheses of many such results (e.g., Reny 1999) fail to be invariant to monotone transformations of payoff functions. Ordinality is achieved by endowing each player with a preference relation over the joint strategy space, and it is not assumed that this relation admits a utility representation. Indeed,

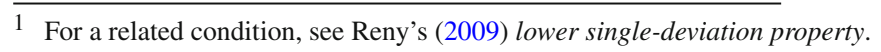


when preferences are discontinuous, the existence of such a representation is not straightforward. An important feature of Reny's hypotheses is that they are entirely local in nature, which makes them easier to check and interpret than otherwise might be the case. In particular, the local conditions are not required to patch together over the entire strategy space in any particular way. Reny's main result, Theorem 3.4, allows the possibility that some players' best- reply correspondences might be well behaved. When this is the case, weaker conditions on the discontinuous payoff functions of the other players are sufficient to ensure the existence of a Nash equilibrium. The resulting theorem, while useful in its own right, provides a new technique for proving equilibrium existence even in discontinuous games that do not directly satisfy its hypotheses. Indeed, one can often handle such a nonconforming game by defining a "bilateral" surrogate game that includes a new player whose strategy space is the entire strategy space and against whom all other players compete. See Reny's Remark $4 .^{2}$ Finally, Reny also shows that the various "correspondence security" ideas first put forward by McLennan et al. (2011) and Barelli and Meneghel (2013), are in fact very much like Reny's $(1999,2009)$ "point security" ideas that they were designed to generalize. Thus, the two ideas are rather more closely related than might at first appear.

The paper by Vincenzo Scalzo (2015) entitled, "Remarks on the existence and stability of some relaxed Nash equilibrium in strategic form games," studies a weakening of Nash equilibrium that he calls quasi-Nash equilibrium. A strategy profile $x^{*}$ is a quasi-Nash equilibrium if $\liminf _{y \rightarrow x^{*}} \sum_{i \in N}\left(u_{i}\left(x_{i}, y_{-i}\right)-u_{i}(y)\right) \leq 0$ for all $x \in X$. Note that if all payoff functions are continuous, then this condition says that $\sum_{i \in N}\left(u_{i}\left(x_{i}, x_{-i}^{*}\right)-u_{i}\left(x^{*}\right)\right) \leq 0$ for all $x \in X$ and so $x^{*}$ is a Nash equilibrium. The aggregator function $\Phi(x, y)=\sum_{i \in N}\left(u_{i}\left(x_{i}, y_{-i}\right)-u_{i}(y)\right)$ plays an important role from the perspective of Fan's $(1953,1972)$ minmax inequality, and its use in Scalzo's paper is inspired by the key role it plays in the paper of Baye et al. (1993). Quasi-Nash equilibria are shown to exist under conditions that do not include any continuity assumptions on payoffs. The main condition that is a required is a form of quasi-concavity of the aggregator function $\Phi(x, y)$. A Nash equilibrium can be shown to exist when appropriate continuity assumptions are added. In particular, as Scalzo observes, the sets of Nash and quasi-Nash equilibria coincide when, in addition, $\Phi(x, y)$ is transfer continuous, i.e., if whenever $\Phi(x, y)>0$, there exists $\hat{x} \in X$ such that $\Phi\left(\hat{x}, y^{\prime}\right)>0$ for all $y^{\prime}$ in some neighborhood of $y$. Transfer continuity can be related Reny's paper in this symposium as follows. Consider a surrogate game between two players, 0 and 1 , each with pure strategy set $X$. Let player 0's payoff function be $H(x, y)=1$ if $x=y$ and $=0$ otherwise, and let player 1's payoff function be $\Phi(x, y)$. Then, this surrogate game is point secure with respect to player set $I=\{1\}$ as defined in Reny's symposium paper if and only if $\Phi(x, y)$ is transfer continuous. Scalzo relates quasi-Nash equilibrium to the concept of Reny equilibrium introduced by Bich and Laraki (2014) who connect the latter equilibrium concept to the endogenous sharing rules of Simon and Zame (1990). Both equilibrium concepts

2 This technique is used in Reny (2015b) to generalize the Schafer and Sonnenschein theorem on the existence of equilibrium in abstract economies. See also the paper by He and Yannelis (2015) in this symposium. 
share the property that they exist so long as a form of quasi-concavity of payoffs holds; in the case of Reny equilibrium, the condition is quasi-concavity itself, i.e., so long as each player's payoff function is own-strategy quasi-concave, a Reny equilibrium exists. Scalzo supplies a number of novel and interesting stability results for both quasi-Nash equilibrium and Reny equilibrium. For example, in Proposition 11, it is shown that within a set of games that have a quasi-Nash equilibrium, there is a dense subset of games such that every quasi-Nash equilibrium of any game in the dense set is essential, i.e., any game with uniformly close payoff functions has a nearby quasi-Nash equilibrium. Such stability properties need not hold for Nash equilibria in discontinuous games and so the results here provide an important motivation for the study of quasi-Nash equilibria and Reny equilibria.

\section{References}

Barelli, P., Meneghel, I.: A note on the equilibrium existence problem in discontinuous games. Econometrica 81, 813-824 (2013)

Baye, M.R., Tian, G., Zhou, J.: Characterizations of the existence of equilibria in games with discontinuous and non-quasiconcave payoffs. Rev. Econ. Stud. 60, 935-948 (1993)

Bich, P., Laraki, R.: On the existence of approximate equilibria and sharing rule solutions in discontinuous games. Cahier du Laboratoire d'Econométrie de 1'Ecole Polytechnique 2014-22, and forthcoming in Theoretical Economics (2014)

Carmona, G.: Reducible equilibrium properties: comments on recent existence results. Econ. Theory (2014). doi:10.1007/s00199-014-0842-y

Carmona, G., Podczeck, K.: Existence of Nash equilibrium in ordinal games with discontinuous preferences. Econ. Theory (2015). doi:10.1007/s00199-015-0901-z

Dasgupta, P., Maskin, E.: The existence of equilibrium in discontinuous economic games, Part I, Theory. Rev. Econ. Stud. 53, 1-26 (1986)

Debreu, G.: A social equilibrium existence theorem. Proc. Nat. Acad. Sci. 38, 886-893 (1952)

Flesch, J., Predtetchinski, A.: Subgame-perfect -equilibria in perfect information games with sigma-discrete discontinuities. Econ. Theory (2015). doi:10.1007/s00199-015-0868-9

Fan, K.: Minimax theorems. Proc. Nat. Acad. Sci. 39, 42-47 (1953)

Fan, K.: A Minimax inequality and applications. In: Shisha, O. (ed.) Inequalities III. Academic Press, New York (1972)

He, W., Yannelis, N.C.: Existence of Walrasian equilibria with discontinuous, non-ordered, interdependent and price-dependent preferences. Econ. Theory (2015). doi:10.1007/s00199-015-0875-X

McLennan, A., Monteiro, P.K., Tourky, R.: Games with discontinuous payoffs: a strengthening of Reny's existence theorem. Econometrica 79, 1643-1664 (2011)

Nessah, R., Tian, G.: On the existence of Nash equilibrium in discontinuous games. Econ. Theory (2015). doi:10.1007/s00199-015-0921-8

Prokopovych, P.: Majorized correspondences and equilibrium existence in discontinuous games. Econ. Theory (2015). doi:10.1007/s00199-015-0874-y

Reny, P.J.: On the existence of pure and mixed strategy Nash equilibria in discontinuous games. Econometrica 67, 1029-1056 (1999)

Reny, P.J.: Further Results on the Existence of Nash Equilibria in Discontinuous Games, Mimeo. University of Chicago, Chicago (2009)

Reny, P.J.: Strategic approximations of discontinuous games. Econ. Theory 48, 17-29 (2010). doi:10.1007/ s00199-0I0-0518-I

Reny, P.J.: Nash equilibrium in discontinuous games. Econ. Theory (2015). doi:10.1007/ s00199-015-0934-3

Reny, P.J.: Equilibrium in discontinuous games without complete or transitive preferences. Econ. Theory Bull. (2015). doi:10.1007/s40505-015-0087-3

Scalzo, V.: Remarks on the existence and stability of some relaxed Nash equilibrium in strategic form games. Econ. Theory (2015). doi:10.1007/s00199-015-0917-4 
Shafer, W.J., Sonnenschein, H.: Equilibrium in abstract economies without ordered preferences. J. Math. Econ. 2, 345-348 (1975)

Simon, L.: Games with discontinuous payoffs. Rev. Econ. Stud. 54, 569-597 (1987)

Simon, L., Zame, W.: Discontinuous games and endogenous sharing rules. Econometrica 58, 861-872 (1990) 